\title{
La evaluación neuropsicológica
}

\section{The neuro-psychological assessment}

\section{Javier TIRAPU USTÁRROZ*}

\section{RESUMEN}

En los últimos años ha existido un aumento de la demanda de exploraciones neuropsicológicas cuyo objetivo se dirige hacia las necesidades de tratamiento que tienen las personas afectadas por alteraciones en las funciones cerebrales superiores. Aquí se van a comentar algunas preguntas generales que conviene plantearse antes de decidir los instrumentos de evaluación que van a ser administrados ¿Son adecuados los contenidos planteados y su nivel de dificultad? ¿Ofrecen información sobre los mecanismos cognitivos alterados subyacentes? ¿Cuál es la validez ecológica de las pruebas? ¿Hasta qué punto estas pruebas son sensibles a los cambios en la situación clínica experimentados por los pacientes? Asimismo, la evaluación de las consecuencias de la lesión cerebral requiere aplicar la observación, la entrevista y diferentes pruebas de evaluación neuropsicológica. Los resultados de esta evaluación representan el punto de partida del tratamiento y rehabilitación neuropsicológica por lo que se revisará los procesos adecuados de evaluación. Para terminar, haremos una breve reseña sobre las dificultades añadidas que entraña la evaluación de las funciones frontales

\section{PALABRAS CLAVE}

Evaluación neuropsicológica, Validez ecológica, Cuantitativo/cualitativo, Neuroimagen, Pruebas especificas, Alteraciones emocionales.

\begin{abstract}
During the last few years there has been an increasing demand of neuro-psychological tests aiming at identifying treatment needs of people with altered high brain functions. Before making the decision about what assessment tools to use, a number of questions

* Neuropsicólogo Clínico. Servicio de Rehabilitación Neurológica. Clínica Ubarmin. Servicio Navarro de Salud. Elcano. Navarra. jtirapu@cfnavarra.es
\end{abstract}


have to be asked. Are the test content and its level of difficulty appropriate? Does the test content provide information about underlying altered cognitive mechanisms? What is the ecological validity of the tests? To what extent are these tests sensitive to changes in the patient's clinical condition? Assessing the consequences of the brain damage requires observation, an interview and a number of neuro-psychological assessment tests. Since the results of this assessment are the starting point for the neuro-psychological treatment and rehabilitation, the assessment process must be checked. Finally, a quick account of difficulties underlying the assessment of frontal functions is given.

\section{KEY WORDS}

Neuro-psychological assessment, Ecological validity, Cuantitative/cualitative, Neorimage, Specific test, Emotional alterations. 


\section{INTRODUCCIÓN}

\subsection{Los objetivos de la evaluación neuropsicológica}

En los últimos años ha existido un aumento de la demanda de exploraciones neuropsicológicas tanto en personas que han sufrido un daño orgánico conocido, como en pacientes con diferentes patologias psiquiátricas en las que existe sospecha de una disfunción cerebral, y es un hecho cada vez más común en la mayoría de los países occidentales la progresiva incorporación de neuropsicólogos clínicos en los servicios hospitalarios. El objetivo fundamental de estas evaluaciones ya no se centra exclusivamente en identificar una posible alteración de las funciones reguladas por la corteza cerebral, sino que se dirige cada vez más hacia las necesidades de tratamiento que tienen las personas afectadas por alteraciones en las funciones cerebrales superiores; lo que permite entender la importancia creciente de los programas de rehabilitación neuropsicológica como un recurso terapéutico cada vez más necesario.

\subsection{Criterios generales a la hora de seleccionar los instrumentos de evaluación}

Una cuestión fundamental que merece ser analizada es el establecimiento de una serie de criterios fundamentales que han de tenerse en cuenta a la hora de seleccionar los instrumentos de evaluación que vamos a emplear. Autores como Wade han establecido una distinción entre criterios mayores y criterios menores. Entre los primeros se incluyen la ética, fiabilidad, validez y sensibilidad a los cambios clínicos, mientras que entre los segundos cabe resaltar la viabilidad de administración, la validación de las pruebas con poblaciones similares, la aplicación de las mismas por diferentes grupos de trabajo y la existencia de protocolos claros y precisos de administración y puntuación.

Aquí se van a comentar algunas preguntas generales que conviene plantearse antes de decidir los instrumentos de evaluación que van a ser administrados, en especial cuando se trata de obtener una información que pueda ser de utilidad en el diseño, implementación y valoración de los programas de tratamiento.

- ¿Son adecuados los contenidos planteados y su nivel de dificultad?

- ¿Ofrecen información sobre los mecanismos cognitivos alterados subyacentes?

- ¿Cuál es la validez ecológica de las pruebas?

- ¿Hasta qué punto estas pruebas son sensibles a los cambios en la situación clínica experimentados por los pacientes?

Adecuación de los contenidos y grado de dificultad. Cuando alguien se enfrenta a la necesidad de realizar una evaluación neuropsicológica trata con personas que conservan un conjunto de habilidades muy diversas en función de sus características personales, topografía de las lesiones, nivel de deterioro, etc. Ello impide hablar de un protocolo rígido de evaluación y de un conjunto de pruebas establecidas de antemano, y exige por contra un nivel de conocimiento que permita determinar en cada caso las pruebas de evaluación más adecuadas.

Así por ejemplo, para alguien con una inteligencia previa superior, un nivel de ejecución dentro de los límites normales en algunas pruebas en las fases iniciales del trastorno no implica necesariamente que no exista deterioro. Y en las fases 
La evaluación neuropsicológica

\section{Tabla 1. Principales objetivos de la evaluación neuropsicológica} (Muñoz, Céspedes, Tirapu, 2001)

- Descripción detallada de las consecuencias de la lesión o disfunción cerebral en términos dc funcionamicnto cognitivo, posibles trastornos dc conducta $\mathrm{y}$ altcracioncs cmocionales.

- Definición de los perfiles clínicos que caracterizan a diferentes tipos de patologías que cursan con deccrioro ncuropsicológico.

- Contribución al cstablecimicnto de un diagnóstico más preciso en decrminadas enlermedades neurológicas y psiquiátricas, en especial en aquellos casos en los que existen alteraciones funcionales no detectadas con las lécnicas de neuroimagen habituales y en los que se encuentran en las primeras etapas de procesos patológicos degencrativos.

- Establccimicnto de un programa de rchabilitación individualizado, a partir del conocimicnto más adecuado de las limilaciones, pero lambién de las habilidades conservadas, que permila optimizar el funcionamicnto independiente y la calidad de vida del paciente.

- Determinación de forma objetiva de los progresos de cada paciente y valoración de la eficacia de los diferentes tratamientos (quirúrgicos, farmacológicos, cognitivos y conductuales).

- Identificación de los factores de pronóstico tanto de la cvolución que cxperimentan las personas afcctadas por alteraciones ncuropsicológicas como del nivel de recuperación que se puede alcanzar a largo plazo.

- Valoración médico-legal del nivel de decrioro cognitivo, que sirva de apoyo a las interpretaciones de testimonios y peritaciones judiciales, y/ o de cara al establecimiento de posibles indemnizaciones.

- Verificación de hipótesis sobre las relaciones entre el cerebro y la conducla, que permita mcjorar nucstra comprensión dcl modo cómo cl ccrcbro proccsa la información.

más avanzadas de la enfermedad, cuando las puntuaciones en los tests cognitivos son minimas, se hace preciso acudir de forma preferente a escalas funcionales que valoren el grado de capacidad de los pacientes. 
Los mecanismos cognitivos alterados. Las pruebas empleadas han de proporcionar información que permita explicar la disminución del rendimiento de un determinado paciente en términos de alteración en una 0 más operaciones 0 componentes de un modelo de funcionamiento cognitivo normal. Los resultados numéricos por sí mismos son de poca utilidad para el establecimiento de las estrategias de rehabilitación más apropiadas para cada caso individual. Se hace necesario un análisis más minucioso tanto de los errores cometidos por los pacientes como de la existencia de posibles formas alternativas de resolver las tareas que se proponen, pues el conocimiento de estos factores resulta de gran interés para el diseño posterior del programa de rehabilitación.

La validez ecológica. La validez ecológica hace referencia a la necesidad de emplear medidas que exploren el grado en que las funciones evaluadas se generalizan a la vida real. Dicho de otro modo, intenta evaluar las relaciones entre los rendimientos del sujeto en un test y la capacidad de funcionar en el mundo real.

En ocasiones las situaciones experimentales de algunas pruebas neuropsicológicas son tan artificiales que los resultados obtenidos tienen muy poco valor a la hora de predecir el nivel de funcionamiento real. Es posible encontrar déficits sutiles que no provocan dificultades en las tareas de vida diaria y viceversa.

Por ello, la tendencia más actual de la evaluación neuropsicológica es desarrollar nuevos instrumentos que exploren conductas y actividades similares a las características del medio natural donde se desarrolla habitualmente la vida de los pacientes.
La sensibilidad al cambio. Algunas de las medidas más utilizadas en la exploración neuropsicológica ofrecen categorias muy generales y poco descriptivas. Apenas informan de la magnitud de los cambios ni de las áreas en que éstos se producen, y ambos aspectos son esenciales tanto en relación con el proceso de rehabilitación como para conocer la evolución del trastorno.

Es necesario escoger pruebas que sean capaces de reflejar los cambios que se producen por el paso del tiempo o con los diferentes tratamientos, con el objeto de ir incorporando nuevos elementos o revisar aquellos objetivos que no se están desarrollando de forma satisfactoria.

\subsection{La polémica cuantitativo/ cualitativo en evaluación neuropsicológica}

Existe un fuerte debate sobre el mejor modo de aproximarnos al estudio de los déficits mostrados por los pacientes -enfoques flexibles y cualitativos versus psicométricos y normativos-, aunque es perfectamente posible integrar ambos planteamientos, como ya se ha puesto de manifiesto en otras áreas de la evaluación psicológica. De hecho, aún cuando en la literatura especializada se ha equiparado con frecuencia estandarizado con cuantitativo y flexible con cualitativo, no resulta justo afirmar que los neuropsicólogos que utilizan pruebas estandarizadas ignoren los datos cualitativos ni que los que defienden los planteamientos flexibles nieguen los criterios cuantitativos. Hay que afirmar que esta distinción se encuentra más estrechamente relacionada con el modo de interpretación de los datos obtenidos a través de los instrumentos de evaluación que con el método por el que se obtienen los mismos. Así, en relación con la rehabilitación neuropsicológica, la aproximación cuantitativa 
puede resulta de utilidad para valorar la eficacia de distintos programas de rehabilitación en grupos de pacientes, mientras que la aproximación más cualitativa permite un análisis más detallado de los errores cometidos por cada paciente y de las estrategias utilizadas y no utilizadas, lo que constituye la base para el establecimiento de un programa de rehabilitación individualizado.

\section{EL PROCESO DE EVALUACIÓN}

La evaluación de las consecuencias de la lesión cerebral requiere aplicar la observación, la entrevista y diferentes pruebas de evaluación neuropsicológica. Los resultados de esta evaluación representan el punto de partida del tratamiento y rehabilitación neuropsicológica.

Tabla 2. La aproximación cuantitativa versus cualitativa en la evaluación neuropsicológica (Muñoz Céspedes, Tirapu, 2001)

\begin{tabular}{|c|c|}
\hline APROXIMACIÓN CUANTITATIVA & APROXIMACIÓN CUALITATIVA \\
\hline $\begin{array}{l}\text { - } \underline{C \Lambda R \Lambda C I R I R I S I C \Lambda S} \\
\checkmark \text { Se centra fundamentalmente en los } \\
\text { resultados que los individuos obtienen en las } \\
\text { prucbas que se les administran. } \\
\checkmark \text { Evaluación orientada al producto. } \\
\checkmark \text { Se trabaja con referencia a normas }\end{array}$ & $\begin{array}{l}\text { - } \underline{C} \text { S R } \Lambda \text { CIFRÍSTICAS } \\
\checkmark \text { Sc precocupa más de analizar cómo lleva a cabo la } \\
\text { persona la tarea que en determinar si la resuelve o no. } \\
\checkmark \text { Evaluación oricntada al proceso. } \\
\checkmark \text { Se trabaja con referencia a un criterio individual }\end{array}$ \\
\hline $\begin{array}{l}\text { - UTIIII) } \Lambda \mathrm{I}) \\
\checkmark \text { Estudios con grupos de pacientes con el } \\
\text { objeto de cncontrar asociaciones de síntomas } \\
\text { comunes. } \\
\checkmark \text { Evaluación de la cficacia de difcrentes } \\
\text { tratamientos o programas de rehabilitación. } \\
\checkmark \text { Valoración del daño corporal. }\end{array}$ & $\begin{array}{l}\text { - U UTILIID } \Lambda \mathrm{I}) \\
\checkmark \text { Estudios de casos para conocer la estructura de los } \\
\text { procesos cognitivos. } \\
\checkmark \text { Rehabilitación neuropsicológica }\end{array}$ \\
\hline $\begin{array}{l}\text { - } \triangle \mathrm{PO} \text { (R' } \Lambda \text { CIONES } \\
\checkmark \text { Exigencia de la necesidad de considerar las } \\
\text { caractcrísticas de fiabilidad y validez de las } \\
\text { pruebas. } \\
\checkmark \text { Mejor categorización diagnóstica de los } \\
\text { pacientes. }\end{array}$ & $\begin{array}{l}\text { - } \triangle \mathrm{PORT} \Lambda \mathrm{CIONES} \\
\checkmark \mathrm{El} \text { análisis de los errores y de las estrategias } \\
\text { utilizadas constituyc la base para cl cstablecimiento } \\
\text { de los programas de rehabilitación. } \\
\checkmark \text { Desarrollo de modelos de procesamiento cognitivo } \\
\text { de la información. }\end{array}$ \\
\hline $\begin{array}{l}\text { QLIMITACIONES } \\
\checkmark \checkmark \text { Facilita cl acceso a la Ncuropsicología de } \\
\text { personas con mínimos conocimientos de la } \\
\text { disciplina. } \\
\checkmark \text { I os datos cuantitativos "per sc" no aportan } \\
\text { nada a la rehabilitación de las personas con } \\
\text { daño cercbral. }\end{array}$ & $\begin{array}{l}\text {-LIMITACIONES (de la Neurop. cognitiva) } \\
\checkmark \text { Hasta ahora nos han dicho muy poco sobre cómo } \\
\text { estos procesos pueden ser modificados a través del } \\
\text { aprendizajc. } \\
\checkmark \text { Ha centrado su atención cxclusivamentc cn los } \\
\text { déficit, olvidando la discapacidad y minusvalía } \\
\text { resultante de las lesiones cercbrales. }\end{array}$ \\
\hline
\end{tabular}




\subsection{La revisión de los informes previos}

Habitualmente el proceso de evaluación comienza con la revisión de los informes previos, cuya información sirve de base para determinar la gravedad y naturaleza de la disfunción, los resultados de las técnicas de neuroimagen, la presencia de complicaciones asociadas, la evolución de la sintomatología y el nivel de dependencia o recuperación alcanzado hasta el momento.

\subsection{La entrevista al paciente y familiares}

La entrevista con el paciente es la siguiente tarea a realizar, y constituye en la mayoria de los casos el instrumento de diagnóstico más poderoso y más económico con el que cuenta el clínico. A través de ella se obtiene información en primer lugar sobre la historia del paciente y sobre variables mediadoras tales como la edad, el nivel de funcionamiento premórbido, el grado de escolaridad y situación laboral alcanzada, etc. Sin un adecuado conocimiento del valor de estas variables mediadoras resultará imposible interpretar ilos resultados de cualquier prueba de evaluación neuropsicológica empleada. Además, la entrevista permite conocer la descripción que hace el paciente de la situación actual, los problemas específicos y la importancia que concede a los mismos, y el grado de autoconciencia de las limitaciones existentes.

Asimismo se debe obtener información sobre los patrones de conducta y personalidad previa para conocer el nivel de ajuste previo y/o descartar la existencia de problemas de inadaptación social (abuso de sustancias tóxicas, déficit de control de impulsos, escasas habilidades sociales, etc.) que puedan contribuir a explicar los problemas emocionales actuales. Finalmente, conviene dedicar una parte de la entrevista a determinar las consecuencias psicosociales que está ocasionando el trastorno en cuestión.

Puede ser conveniente entrevistar de forma independiente al paciente y a los familiares. Son numerosos los trabajos que han evidenciado diferencias significativas entre la información proporcionada por los familiares y por los afectados con lesiones cerebrales (por ejemplo, es bien conocido que las personas con traumatismos craneoencefálicos graves tienden a sobreestimar su nivel de competencia cognitiva y conductual, mientras que suele suceder lo contrario después de un daño cerebral leve). Por lo tanto, el motivo de estas entrevistas es doble: por un lado permiten obtener una información lo más completa posible del estado del paciente, de su evolución y de los cambios psicosociales acaecidos, pero además nos permiten contrastar el grado de desacuerdo entre las dos fuentes, lo que constituye un elemento esencial para conocer el grado de autoconciencia que tiene la persona con daño o disfunción cerebral de sus propias limitaciones.

\subsection{Las técnicas de neuroimagen}

El estudio del sistema nervioso central y de los procesos cognitivos se acompaña de un conjunto de técnicas de exploración complementaria: análisis de parámetros neuroquímicos, registros electrofisiológicos (EEG, potenciales evocados) y técnicas de neuroimagen. En especial, estas últimas han evolucionado con una rapidez vertiginosa y han supuesto una auténtica revolución en el diagnóstico neurorradiológico. Las técnicas más modernas pueden clasificarse en dos grandes grupos, en función del tipo de información que ofrecen: 
1. Estudio de la anatomía y estructura cerebral (neuroimagen estructural):

- TAC (Tomografía axial computarizada)

- RMN (Resonancia magnética nuclear)

2. Estudio de la fisiología y función cerebral (neuroimagen funcional):

- SPECT (Tomografía por emisión de fotón único)

- PET (Tomografia por emisión de positrones)

- RMNf (Resonancia magnética nuclear funcional)

Las técnicas de neuroimagen estructural son de gran utilidad para la detección y localización de la lesión (por ejemplo, tumores cerebrales), al mismo tiempo que proporcionan una línea base que permite comparar y controlar diferentes trastornos patológicos agudos y subagudos (por ejemplo, la absorción o expansión del edema o hematomas cerebrales después de un T.C.E.). Una vez superada la fase aguda, la RMN constituye la técnica de elección para el seguimiento y control posterior, al ofrecer una mayor resolución que permite detectar lesiones pequeñas o difusas que pueden pasar desapercibidas pero que resultan esenciales para explicar las alteraciones neuropsicológicas que muestran los pacientes con un daño cerebral.

Las técnicas de neuroimagen funcional registran la perfusión sanguínea regional y el metabolismo de la glucosa o el consumo de oxígeno, lo que supone un indicador más directo de la actividad cerebral La idea central para entender su funcionamiento es que tanto el flujo san- guíneo como el metabolismo aumentan de forma proporcional al incremento de la actividad en una determinada región cerebral; por lo tanto, la presencia de anomalías o déficit en estos parámetros sugieren la existencia de áreas de disfunción cerebral. La aparición de estas nuevas técnicas está desplazando la preferencia del método clásico de la neurología conductual del análisis de las lesiones por una metodología de registro funcional. Ello se explica por varias razones: a) en el ámbito de la investigación básica permiten determinar con más precisión las estructuras cerebrales y redes neurales que se relacionan con un proceso cognitivo particular (por ejemplo, atención o lenguaje); b) en tareas de evaluación y diagnóstico clínico, ofrecen correlaciones más precisas entre las alteraciones funcionales cerebrales objetivadas por las mismas y las alteraciones cognitivas y conductuales que persisten después de un posible daño cerebral, y c) en el proceso de rehabilitación y seguimiento de los resultados del tratamiento, presentan una mayor sensibilidad para registrar cambios en la activación de diferentes áreas cerebrales después de una intervención farmacológica o conductual, que no son detectadas con las técnicas estructurales.

\subsection{La evaluación neuropsicológica formal}

De forma general, la valoración de las personas con una disfunción cognitiva incluye, al menos, el estudio del rendimiento intelectual general, la atención, la velocidad de procesamiento de la información, la capacidad de aprendizaje y memoria, las habilidades perceptivas y motoras, el lenguaje y la comunicación, el razonamiento, la capacidad de solución de problemas y otras funciones asociadas a los lóbulos frontales. A continuación se agrupan los instrumentos de 
evaluación en tres grandes grupos, sin pretender realizar una descripción exhaustiva de las pruebas neuropsicológicas, si no señalar exclusivamente algunas características generales de cada uno de los tipos de pruebas. El lector interesado en un análisis exhaustivo de los diferentes tests puede consultar las obras monográficas de Spreen y Strauss (26) y Lezak (27).

- Escalas breves o pruebas de rastreo cognitivo.

- Baterías neuropsicológicas generales.

- Tests específicos.

Escalas breves o pruebas de rastreo cognitivo. Son tests de fácil aplicación y que requieren poco tiempo para su aplicación (de cinco a veinte minutos). Constan de un conjunto de preguntas variadas en relación con un cierto número de áreas cognitivas (orientación temporal y espacial, atención y concentración, aprendizaje y memoria, lenguaje, capacidades visoespaciales, etc.). La mayoría se diseñaron originalmente para la cuantificación de los déficit cognitivos en pacientes ancianos, aunque se han aplicado de forma general a pacientes con todo tipo de déficit cognitivos tanto agudos como crónicos. La puntuación global obtenida permite obtener un "punto de corte" que se utiliza como una distinción dicotómica entre normal y patológico, señalando aquellos individuos que precisan de una evaluación neuropsicológica más detallada. Una limitación importante es la falta de sensibilidad para detectar déficit cognitivos focales. Por ejemplo pacientes con lesiones frontales o con lesiones en el hemisferio derecho pueden conseguir puntuaciones máximas en la mayoría de los ítems. Además su especificidad es muy baja, incluso en los casos en que se valora el perfil de las diferentes secciones de los tests.

Por lo tanto, sus principales utilidades se limitan a proporcionar una visión rápida del paciente (por ejemplo, en el contexto de una consulta de neurología o psiquiatria general, servir de guia para valorar el seguimiento de los pacientes a lo largo del tiempo y establecer correlaciones entre esta puntuación global y otras variables relevantes.

Baterías generales de evaluación. De forma genérica, se puede definir una batería de evaluación neuropsicológica como un conjunto de pruebas o elementos que exploran las principales funciones cognitivas de forma sistematizada, con el objeto de detectar y tipificar la existencia de un daño cerebral.

Existe una gran controversia en la literatura especializada sobre las utilidades y limitaciones de la aplicación de este tipo de procedimientos en la evaluación de los pacientes con lesión o posible disfunción cerebral. Las principales ventajas de su uso radican en la posibilidad de estudiar los principales sindromes y alteraciones neuropsicológicas en un tiempo relativamente breve, la oportunidad de disponer de una amplia base de datos que facilita, por un lado, la obtención de perfiles que caracterizan a diferentes lesiones cerebrales (por ejemplo, accidentes isquémicos o hemorragias en diferentes territorios vasculares), y por otro, un mayor control sobre un conjunto de variables (edad, nivel educativo, etc.) que afectan al rendimiento de los individuos en estas pruebas. Finalmente, al realizar una valoración global del funcionamiento cognitivo permiten identificar no sólo los principales déficit sino también las habilidades preservadas en cada paciente. Entre los inconvenientes cabe señalar la falta de fundamentación teórica, pues en general constituyen una 
agrupación de pruebas más o menos sensibles a los efectos de diferentes lesiones cerebrales, pero carecen de un marco conceptual que explique dicha selección de instrumentos. Y en el ámbito clínico, su diseño permite más la comparación de resultados entre individuos y grupos que el análisis específico de los errores que comete cada paciente, lo cual resulta esencial para el establecimiento de un posterior programa de rehabilitación neuropsicológica personalizado.

Pruebas específicas de evaluación neuropsicológica. Resulta imposible establecer un protocolo estandarizado para la exploración neuropsicológica, ya que la selección de las pruebas viene condicionada no sólo por su sensibilidad a la detección del daño cerebral, sino también por el motivo que determina la valoración -diagnóstico, -, por el estado cognitivo de cada paciente y por el tiempo disponible para realizar el estudio. Y en el ámbito que nos ocupa, el de la rehabilitación neuropsicológica, la información que se requiere difiere mucho en función del contexto terapeútico en que se trabaja. Así por ejemplo, en una unidad de rehabilitación hospitalaria las principales cuestiones a las que ha de responder la evaluación se pueden centrar en examinar la capacidad del paciente para seguir instrucciones o recordar las habilidades entrenadas en las sesiones de fisioterapia. Por el contrario, para el profesional que trabaja en un centro de dia, los mismos datos se pueden utilizar para ayudar a determinar la seguridad del paciente en la comunidad o para colaborar con el monitor laboral en la elaboración de un plan de trabajo realista para el paciente. Todo ello condicionará la naturaleza y contenido de los instrumentos de evaluación empleados.

En lo que sí existe un mayor consenso es en la necesidad de: a) realizar una exploración detallada de las principales funciones cognitivas, dado el carácter difuso y multifocal de las lesiones cerebrales y la heterogeneidad de las alteraciones neuropsicológicas que pueden persistir en estos pacientes.

b) recoger información que nos permita obtener un perfil de los déficit pero también de las capacidades preservadas de los pacientes, ya que éstas son también de gran relevancia para la elaboración del programa de rehabilitación neuropsicológica.

c) seleccionar procedimientos que se muestren sensibles ante los cambios que se producen a lo largo del proceso rehabilitador y que tengan validez ecológica; es decir que las funciones y conductas evaluadas guarden relación con las actividades que desarrollan los pacientes en su vida cotidiana.

Una cuestión especialmente problemática es la evaluación de los déficits en el funcionamiento ejecutivo. Se han propuesto diferentes pruebas que han mostrado su utilidad y sensibilidad para detectar una disfunción de las áreas prefrontales (Trail Making Test, test de clasificación de cartas de Wisconsin, Torre de Hanoi, etc.). Sin embargo diferentes trabajos desarrollados en la última década han puesto de relieve que algunos pacientes con lesiones prefrontales bien identificadas realizan bien estas pruebas neuropsicológicas, aún cuando presentan dificultades notables en su adaptación a la vida cotidiana.

Esta limitación de las pruebas de evaluación neuropsicológica nos sitúa ante una nueva demanda, que resulta más pertinente si cabe cuando el objetivo que 
Tabla 3. Pruebas específicas de evaluación neuropsicológica

\begin{tabular}{|c|c|}
\hline $\begin{array}{l}\text { Orientación temporal } y \\
\text { espacial }\end{array}$ & $\begin{array}{l}\text { - } \quad \text { Prueba de orientación de Christensen } \\
\text { - Subtests del Test Barcelona }\end{array}$ \\
\hline $\begin{array}{l}\text { Atención/concentración/velo } \\
\text { cidad de procesamiento de } \\
\text { la información }\end{array}$ & $\begin{array}{l}\text { - } \quad \text { Continuous Performance Test (C.P.T.) } \\
\text { - } \quad \text { Retención de dígitos } \\
\text { - Trail making Test (forma A y B) } \\
\text { - } \quad \text { Clave de números del WAIS III } \\
\text { - Test de atención breve (B.T.A.) } \\
\text { - Paced Auditory Serial Adition } \\
\text { (P.A.S.A.T.) }\end{array}$ \\
\hline $\begin{array}{l}\text { Habilidades visoespaciales } \\
\text { y visoconstruccionales }\end{array}$ & $\begin{array}{l}\text { - Test de organización visual de Hooper } \\
\text { - Test de orientación de líneas } \\
\text { - Test de reconocimiento de caras } \\
\text { - } \quad \text { Test de retención visual de Benton } \\
\text { - } \quad \text { objetóa de tests para la percepción visual de } \\
\text { - Rompecabezas y cubos del WAIS III }\end{array}$ \\
\hline $\begin{array}{l}\text { Praxias, coordinación y } \\
\text { velocidad manual }\end{array}$ & $\begin{array}{l}\text { - } \text { Finger Tapping Test } \\
\text { - } \quad \text { Prooved Pegboard Test } \\
\text { - Tardue Pegboard Test } \\
\text { - } \quad \text { Pruebas de praxias de Christensen } \\
\text { - Test de apraxia de Goodglass y Kaplan }\end{array}$ \\
\hline Memoria & $\begin{array}{l}\text { - } \quad \text { Escala de memoria de Wechsler revisada } \\
\text { - } \text { Figura compleja de rey } \\
\text { - Test de aprendizaje verbal de Rey } \\
\text { - } \text { Test de aprendizaje verbal de California } \\
\text { - Cuestionario de memoria autobiográfica } \\
\text { (A.M.I.) }\end{array}$ \\
\hline Lenguaje y comunicación & $\begin{array}{l}\text { - } \quad \text { Test de denominación de Boston } \\
\text { - Subtests del test Barcelona } \\
\text { - } \quad \text { Test para la evaluación de la afasia de Boston } \\
\text { Evaluación del procesamiento lingüístico en la } \\
\text { afasia (E.P.L.A.) } \\
\text { Porch Index of Communicative Ability } \\
\text { (P.I.C.A.) }\end{array}$ \\
\hline Razonamiento & $\begin{array}{l}\text { - Comprensión, semejanzas, historietas del } \\
\text { WAIS III } \\
\text { - Test de formación de conceptos de } \\
\text { - Hanfmann-Kasanin } \\
\text { - Test de categorías de Halstead-Reitan }\end{array}$ \\
\hline Funciones ejecutivas & $\begin{array}{l}\text { - } \quad \text { Trail Making Test (forma B) } \\
\text { - } \quad \text { Test de Stroop } \\
\text { - } \quad \text { Fluidez verbal fonética (F.A.S.) } \\
\text { - } \quad \text { Test de de diseños } \\
\text { (W.C.S.T.) } \\
\text { - Test de las torres (Hanoi, Londres). }\end{array}$ \\
\hline
\end{tabular}


se persigue la planificación, puesta en marcha 0 valoración de la eficacia de los programas de rehabilitación. Se hace necesario complementar el empleo de pruebas especificas con:

a) La utilización de medidas que permitan obtener información sobre otros aspectos fundamentales del comportamiento humano como la motivación y la emoción.

b) El desarrollo de sistemas de observación y hojas de registro de conductas en el medio natural, en ambientes no protegidos, ante tareas no propuestas de forma explícita por el evaluador y en situaciones novedosas imprevistas y cambiantes.

\section{LA EVALUACIÓN DE LAS ALTERACIONES EMOCIONALES}

Cuando se explora a una persona con sospecha de disfunción cerebral o lesión ya confirmada hay que preocuparse también por evaluar los cambios emocionales y de personalidad, porque las alteraciones en el funcionamiento cerebral no afectan de forma exclusiva a la cognición, sino también a la capacidad de comprensión, expresión y vivencia de la experiencia emocional (por ejemplo, labilidad emocional, trastornos bipolares, etc.), así como a la propia estructura de la personalidad (por ejemplo, trastorno orgánico de la personalidad. Por otro lado no es menos cierto que la personalidad previa de cada individuo interactúa con el propio trastorno neurológico o neuropsiquiátrico, y su conocimiento permite comprender mejor el modo en que cada paciente afronta los déficit tanto físicos como cognitivos ocasionados por la alteración en el funcionamiento cerebral.

Así, por ejemplo, las conductas de oposición o rechazo que muestran algu- nos pacientes ante la intervención neuropsicológica, logopédica o de fisioterapia han de ser cuidadosamente analizadas. Resulta esencial en estos casos un análisis funcional que permita determinar si el paciente es consciente y asume sus limitaciones actuales, si sus accesos de irritabilidad o ira están relacionados con la dificultad para modular la expresión de las emociones, si está respondiendo a una equivocada percepción del contexto emocional de la situación, o si constituye una manifestación clínica de un estado afectivo alterado (por ej. depresión). Es evidente que el tipo de intervención que se propone en cada uno de estos casos será bien diferente (incrementar la conciencia de los déficits y de las limitaciones que conllevan, empleo de técnicas de autocontrol, tratamiento del problema depresivo subyacente...).

Mientras que existen en el mercado una gran cantidad de pruebas destinadas a la evaluación de los déficit cognitivos, son escasos los instrumentos de evaluación que permiten estudiar de forma específica las alteraciones emocionales que se observan en estas personas. La información sobre estos problemas procede en la mayoría de los casos de los datos proporcionados durante la entrevista por el paciente 0 sus acompañantes 0 , en el mejor de los casos, por las observaciones registradas por el propio psicólogo u otros profesionales. En otras ocasiones se acude a test e inventarios diseñados para otras alteraciones psicopatológicas y cuyo empleo en pacientes con daño cerebral puede resultar problemático e inducir a confusión Un buen ejemplo que permite corroborar esta afirmación lo constituye el empleo de la prueba MMPI, que ofrece en personas con disfunción cerebral unos resultados muy poco consistentes e incluso contradictorios.

No obstante, pese a estas limitaciones 
en los últimos años se ha incrementado el interés por el estudio de los trastornos emocionales después de una disfunción cerebral y se han elaborado diferentes pruebas específicas para el estudio de las alteraciones conductuales de estos pacientes, como son la escala de registro neuroconductual revisada, la escala conductual general, la entrevista de Iowa 0 el inventario de funcionamiento neurocomportamental, o algunas otras escalas para el estudio de las alteraciones depresivas que aparecen después de accidentes cerebrovasculares. Junto a estas pruebas pueden resultar útiles algunos cuestionarios generales de afrontamiento, que informan sobre la presencia o no de estrategias para resolver los problemas, la existencia de una actitud activa/pasiva, reflexiva/impulsiva... frente a las dificultades. No hay que olvidar que algunas personas -sobre todo aquellas que tienen déficits cognitivos leves y una buena conciencia de las limitaciones que ocasionan- tienden a evitar enfrentarse a situaciones en las que su nivel de competencia pueda ser puesto en entredicho. En estos casos, la enseñanza de estrategias compensatorias que minimicen las limitaciones puede resultar insuficiente, si no se garantiza la adopción de un estilo de afrontamiento más activo frente a las distintas situaciones problemáticas que de forma inevitable van surgir.

\section{UNA MIRADA A LO FUNCIONAL}

La necesidad de una orientación más ecológica en la evaluación neuropsicológica ha puesto en el énfasis en que junto a la identificación de los principales déficits cognitivos resulta esencial la valoración del impacto que estos problemas generan en los aspectos funcionales de la vida diaria y la determinación de la capacidad que tiene la persona para cuidar de sí mismo y para llevar a cabo una vida ocupacional y socialmente activa.
Por un lado, se vienen desarrollando diferentes pruebas especialmente diseñadas para estudiar los procesos cognitivos en situaciones de la vida diaria. Se pueden citar, entre otras, el test de competencia cognitiva, el test de atención cotidiana, el test conductual de memoria Rivermead, el test de evaluación conductual del sindrome disejecutivo o diferentes escalas para valorar las habilidades de comunicación (Test de las capacidades de comunicación en la vida diaria, perfil del deterioro funcional de la comunicación, etc.).

Por otra parte, se incluye cada vez con más frecuencia, la evaluación del nivel de independencia funcional. Existe en el mercado un conjunto de pruebas basadas en la observación del paciente en situaciones de la vida real, que recogen información sobre la capacidad de los individuos para realizar diferentes actividades de la vida diaria básicas (autocuidado y movilidad), instrumentales (empleo de medios de transporte, utilización de dinero, manejo de la medicación, etc.) y avanzadas (aficiones, participación en redes sociales...). Pero además es preciso recabar información sobre el nivel de apoyo familiar y social disponible y sobre el grado de conciencia de las limitaciones que muestran tanto el paciente como las personas que le rodean, ya que estos dos elementos modulan el nivel de competencia cognitiva y conductual que puede alcanzar la persona a través de cualquier programa de rehabilitación.

La adecuada valoración de estos aspectos cobra una especial relevancia en relación con los objetivos de la rehabilitación neuropsicológica ya que:

a) Permite adaptar el tipo de tareas que se emplean a las dificultades y necesidades específicas de cada individuo en el contexto cotidiano, 
La evaluación neuropsicológica

Tabla 4. Relevancia de los déficits cognitivos para la realización de las actividades de la vida cotidiana

\begin{tabular}{|c|c|}
\hline DÉFICIT & LIMITACIÓN FUNCIONAL \\
\hline $\begin{array}{l}\text { Reconocimiento esquema } \\
\text { corporal }\end{array}$ & $\begin{array}{l}\text { Dificultades para: } \\
\text { - Vestirse } \\
\text { - Utilizar objetos en relación con el cuerpo (empleo } \\
\text { del peine, cepillo de dientes, cubiertos...) }\end{array}$ \\
\hline Atención sostenida & - Dificultad para seguir una película \\
\hline Atención selectiva & $\begin{array}{l}\text { - Incapacidad para trabajar en un entorno } \\
\text { otras personas, ruidos, etc. }\end{array}$ \\
\hline Atención dividida & $\begin{array}{l}\text { Dificultades para: } \\
\text { - Conducir un vehículo } \\
\text { - Cuidado del hogar/hijos }\end{array}$ \\
\hline Memoria semántica & $\begin{array}{l}\text { Olvido de: } \\
\text { - Conocimientos previamente adquiridos } \\
\text { - Nombre de personas conocidas }\end{array}$ \\
\hline Memoria episódica & $\begin{array}{l}\text { Olvido de: } \\
\text { - Lugar donde se ha aparcado el coche o donde } \\
\text { han dejado las llaves, gafas... } \\
\text { - Itinerarios realizados en las últimas vacaciones }\end{array}$ \\
\hline Memoria prospectiva & $\begin{array}{l}\text { - Las citas que se tienen al dia siguiente } \\
\text { - Los objetos, alimentos... que se han de comprar }\end{array}$ \\
\hline Funciones ejecutivas & $\begin{array}{l}\quad \text { Dificultades para: } \\
\text { - Planificar y realizar la comida } \\
\text { - Utilizar el ordenador } \\
\text { - } \text { Controlar los gastos del mes } \\
\text { - Planificar un viaje } \\
\text { - Resolver una situación problemática } \\
\text { ejemplo, haber perdido el tren) }\end{array}$ \\
\hline
\end{tabular}

lo que constituye la principal garantía para la puesta en marcha y generalización de los logros obtenidos en las sesiones de rehabilitación. b) Hace posible ofrecer a los familiares y cuidadores asesoramiento, con el objeto de ayudar en la resolución de problemas específicos y en su prevención antes de que sucedan. 
c) Constituyen los indicadores más adecuados para valorar la eficacia de los programas de rehabilitación (análisis de los resultados).

\section{LA EVALUACIÓN DE LOS RESULTADOS DE LOS PROGRAMAS DE REHABILITACIÓN}

La última cuestión planteada en el epigrafe anterior merece un comentario más detallado. La evaluación no tiene como finalidad exclusiva la identificación de de los déficits neuropsicológicos, sino que también ofrece información sobre el grado de cumplimiento alcanzado en relación con los objetivos fijados. Esta información resulta esencial desde el punto de vista científico para identificar de forma más precisa qué tipos de estrategias son más eficaces en rehabilitación neuropsicológica (intervenciones basadas en la evidencia). Pero además, desde la perspectiva de gestión de recursos y servicios de salud es cada vez más importante conocer la relación coste/beneficio de estos programas, para valorar si la inversión en este tipo de programas está justificada y organizar la provisión de servicios sanitarios y sociales a las personas afectadas por este tipo de patologias. Es decir, la evaluación de los programas de intervención ha de ofrecer respuesta a tres cuestiones diferentes: el análisis de los diseños de intervención, el control del desarrollo del programa y la valoración de su utilidad, es decir de su eficacia y eficiencia.

En el ámbito de la rehabilitación neuropsicológica demostrar la eficacia de las intervenciones es más complejo que en otras áreas, en la medida que los programas han de concentrarse más en la discapacidad y en la minusvalia ocasionada por el daño o disfunción que en la propia restitución de los déficits. Existe una fuerte controversia sobre el tipo de medi- da más adecuado para valorar el resultado de un programa de rehabilitación, pues es evidente que no existe un indicador único y objetivo de éxito y que resulta necesario incluir medidas objetivas (por ej. Integración laboral, capacidad para vivir de forma independiente...) con otros de naturaleza más subjetiva (calidad de vida, grado de satisfacción de los usuarios y familiares). En lo que sí existe acuerdo es en señalar que la diferencia pre/post en las pruebas de evaluación neuropsicológica no constituyen un criterio suficiente que garantice la efectividad de nuestra intervención. Es evidente que la mejoría de las puntuaciones en las pruebas neuropsicológicas (por ejemplo, Trail Making Test, escala de aprendizaje verbal de California) no constituyen en ningún caso un objetivo de la intervención. Por el contrario, en otros casos, son fácilmente detectables los progresos en la situación clínica del paciente, aunque las diferencias en los resultados de las pruebas neuropsicológicas no alcancen el nivel de significación estadística.

En este sentido, las pruebas de evaluación funcional y otras escalas de valoración de las consecuencias psicosociales representan un tipo de acercamiento más cercano a las necesidades y demandas cotidianas que plantean tanto los pacientes como las personas con las que conviven (capacidad para vivir de forma independiente, integración social, participación en actividades de ocio o productivas...), y que ofrecen una respuesta más satisfactoria para valorar el grado de cumplimiento de los objetivos. Así, en los últimos 10 años, de acuerdo con los conceptos de discapacidad y minusvalía recogidos por la O.M.S. se han desarrollado diferentes medidas y protocolos de evaluación tales como la Medida de evaluación Funcional (F.A.M.), la escala de necesidad de supervisión (Supervision Rating Scale -S.R.S-),el cuestionario de Integración Comunitaria (C.I.Q), el infor- 
me sobre evaluación de la discapacidad (Craig Handicap Assessment and Reporting Technique -C.H.A.R.T.-. Incluso autores como Malec en Estados Unidos han empezado a proponer métodos generales de evaluación y análisis para valorar el grado de cumplimiento de los objetivos en relación con los programas de rehabilitación neuropsicológica (Goal Attainment Scaling in Rehabilitation -G.A.S.-). Se necesita aún más investigación para determinar cuáles de estos protocolos ofrecen una mayor sensibilidad y capacidad de discriminación ante los cambios, tienen una mayor validez de pronóstico y resultan más prácticos y fáciles de aplicar de forma rutinaria, pero el camino ya ha sido iniciado.

\section{SOBRE LA VALIDEZ ECOLÓGICA DE LA EVALUACIÓN DE LAS FUNCIONES FRONTALES}

Como señala Mesulam (1986) "la evaluación de los cambios conductuales asociados con lesiones del córtex prefrontal introduce dificultades adicionales

Tabla 5. Medida de evaluación funcional

(Functional Assessment Measure -F.A.M.- Hall et al, 1992)

\section{Escala:}

7- Independencia completa

6- Independencia modificada (tiempo extra, equipamiento)

5- Supervisión (dirección)

4- Ayuda mínima (realiza más del $75 \%$ de la tarea)

3- Ayuda moderada (50-74\% de la tarea)

2- Ayuda máxima (25-49\% de la tarea)

1- Asistencia total

\begin{tabular}{|l|l|l|l|}
\hline & INICIAL & OBJETIVOS & REVISIÓN \\
\hline AUTOCUIDADO & & & \\
- Deglución & & & \\
- Alimentación & & \\
- Bspecto personal & & \\
- Vestido parte superior & & \\
- Restido parte inferior & & \\
Retrete & & \\
- ConTROL DE ESFÍNTERES & & \\
- Control intestinal & & & \\
\hline
\end{tabular}


Tabla 5. Medida de evaluación funcional (Functional Assessment Measure -F.A.M.- Hall et al, 1992) (Continuación)

\section{MOVILIDAD}

- Transferencias silla/silla de ruedas

- Transferencias retrete

- Transferencias bañera/ducha

- Transferencias coche

- Deambulación (caminar, empleo de silla de ruedas)

- Escaleras

- Desplazamientos por la comunidad

COMUNICACIÓN

- Comprensión

- Expresión verbal/no verbal

- Lectura

- Escritura

- Habla

\section{AJUSTE PSICOSOCIAL}

- Relación social

- Estado de ánimo

- Ajuste a las limitaciones

- Empleo

\section{FUNCIÓN COGNITIVA}

- Resolución de problemas

- Memoria

- Orientación

- Atención

- Seguridad

\section{OTRAS CATEGORIAS}

- Independencia doméstica

- Independencia ocupacional 
ya que estos cambios son excesivamente complejos, variables, dificiles de definir en términos técnicos e imposibles de cuantificar con los test disponibles en la actualidad".

Una cuestión especialmente problemática es la evaluación de los déficits en las denominadas funciones ejecutivas. Las funciones ejecutivas han sido definidas como los procesos que asocian ideas, movimientos y acciones simples y los orientan a la resolución de conductas complejas. Luria (1964) fue el primer autor que sin nombrar el término conceptualizó las funciones ejecutivas como una serie de trastornos en la iniciativa, en la motivación, en la formulación de metas y planes de acción y en la automonitorización de la conducta asociado a lesiones frontales. El término de funciones ejecutivas es debido a Muriel Lezak $(1982,1987)$ que las define como las capacidades mentales esenciales para llevar a cabo una conducta eficaz, creativa y aceptada socialmente. A su vez, Sholberg (1989) considera que las funciones ejecutivas abarcan una serie de procesos cognitivos entre los que destacan la anticipación, elección de objetivos, planificación, selección de la conducta, autorregulación, automonitorización y uso de feedback. Mateer ( citada por Junqué) (1994) continuando con esta linea cognitivista refiere los siguientes componentes de la función ejecutiva: dirección de la atención, reconocimiento de los patrones de prioridad, formulación de la intención, plan de consecución o logro, ejecución del plan y reconocimiento del logro. Como se puede observar en la definición del contenido de estas funciones, el concepto no dista mucho de lo que entendemos por inteligencia.

Como se ha señalado anteriormente, las alteraciones en las funciones ejecutivas se han considerados prototipicas de la patología del lóbulo frontal, fundamentalmente de las lesiones o disfunciones que afectan a la región prefrontal dorsolateral. Así se ha acuñado el término sindrome disejecutivo para definir las dificultades que exhiben algunos pacientes con una marcada dificultad para centrarse en la tarea y finalizarla sin un control ambiental externo. En segundo lugar presentan dificultades en el establecimiento de nuevos repertorios conductuales y una falta de habilidad para utilizar estrategias operativas. En tercer lugar muestran limitaciones en la productividad y creatividad con falta de flexibilidad cognitiva. En cuarto lugar la conducta de los sujetos afectados por alteraciones en el funcionamiento ejecutivo pone de manifiesto una incapacidad para la abstracción de ideas mostrando dificultades para anticipar las consecuencias de su comportamiento, lo que provoca una mayor impulsividad o incapacidad para posponer una respuesta. Dada la múltiplicidad de manifestaciones de este sindrome disejecutivo parece necesario distinguir las funciones ejecutivas de aquellas que no lo son, con el fin de establecer una taxonomía funcional que nos permita distinguir las ejecuciones, habilidades y conductas que son características de un adecuado funcionamiento ejecutivo.

Desde el punto de vista neuroanatómico, se han descrito diferentes circuitos funcionales dentro del córtex prefrontal. Por un lado, el circuito dorsolateral se relaciona más con actividades puramente cognitivas como la memoria de trabajo, la atención selectiva, la formación de conceptos o la flexibilidad cognitiva. Por otro lado, el circuito ventromedial se asocia con el procesamiento de señales emocionales que guian nuestra toma de decisiones hacia objetivos basados en el juicio social y ético. El córtex prefrontal, pues, debe considerarse como un área de asociación heteromodal interconecta- 
da con una red distribuida de regiones corticales y subcorticales.

Para valorar estos déficits ejecutivos se han propuesto múltiples pruebas 0 test neuropsicológicos que han mostrado, en lineas generales, su utilidad para detectar disfunciones del córtex prefrontal como el Wisconsin Card Sorting Test (Heaton, Chelune, Talley, 1993) Stroop (Goleen, 1974), Trail Making Test (Jarvis, Barth, 1987), Fluidez Verbal Fonética (Thurstone, 1962), Fluidez de diseños (Jones-Guttman, 1977), Test de las Torres (Shallice,1982) , etc.

Mientras estos test han mostrado alguna sensibilidad para captar disfunción cerebral frontal, ninguno de ellos ha probado ser específico para medir disfunciones del sistema ejecutivo. Así, algunos pacientes con daño cerebral frontal ejecutan adecuadamente estas pruebas mientras otros pacientes con lesiones retrorolándicas los pueden ejecutar de forma inadecuada a lo que hemos de añadir la considerable variabilidad en la ejecución entre controles normales.

Sin embargo, desde que en 1985 Eslinger y Damasio (Eslinger P.J:, Damasio A 1985, Saver J.L:, Damasio A.R.1991) publicaron el conocido caso de EVR ha quedado patente que algunos pacientes con lesiones prefrontales pueden ejecutar las pruebas neuropsicológicas dentro de limites de normalidad a lo que añadiriamos el trabajo de Anderson, Damasio, Jones y Travel (1991) donde demostraron la falacia de la solidez de la relación existente entre la ejecución en los test neuropsicológicos y la localización de la lesión. Examinaron 91 pacientes mediante RNM y TAC verificando lesiones cerebrales focales ( 49 frontales, 24 no frontales y 18 con lesiones difusas) no encontrando diferencias significativas entre grupos en la ejecución del WCST.
Lo reseñado anteriormente debe llevar a plantearnos que cada paciente debe ser tratado como un caso único que requiere una explicación independiente, es decir, cada uno de ellos representaría un test independiente de la teoría cognitiva (Ellis, Young, 1991). Por otro lado hemos de reconocer la existencia de graves problemas para medir las funciones ejecutivas como son la complejidad de la estructura y funcionamiento del lóbulo frontal, la poca operatividad de la descripción de funciones ejecutivas, la estructura de los test y de la situación de pasación de pruebas y por último el peso que se le concede en la evaluación a lo cuantitativo y no tanto a los procesos de resolución implicados ( no deberíamos olvidar que un test tiene como cometido provocar una conducta que, se supone, tiene su traducción en le funcionamiento cotidiano del individuo).

En lo referente a la situación artificial de la pasación de pruebas Acker (1990) plantea una serie de diferencias entre esta situación de laboratorio y la vida real: en la primera situación la estructura es dada por el examinador, se centra en tareas concretas, el ambiente no es punitivo, la motivación es aportada por el examinador, se da cierta persistencia del estímulo, no se enfatiza el fracaso, el ambiente es protegido y la competencia ausente. En la vida cotidiana es frecuente enfrentarse a tareas no estructuradas y espontáneas, la planificación es individual, la automotivación resulta necesaria, el estímulo no es persistente, se da cierto temor al fracaso, el medio se encuentra menos protegido y existe competencia.

Todo lo anteriormente expuesto nos lleva a plantearnos la validez ecológica de los test neuropsicológicos que miden las funciones ejecutivas ya que esta validez ecológica esta mediatizada por algunas premisas de gran relevancia como 
son: debe existir una relación funcional y predictiva entre ejecución en test y conducta del paciente en situaciones de la vida real, debemos asumir que las demandas son variadas e idiosincráticas resultado de su naturaleza específica, la interacción entre demanda y recursos del paciente puede compensar o exacerbar sus déficits, las condiciones en la pasación de test son determinantes para plantear la generalización, los resultados obtenidos en los test pueden generar falsas expectativas en cuanto al funcionamiento del sujeto en la vida real, la interacción examinador-paciente puede condicionar los resultados, los protocolos utilizados y la extensión y complejidad de los puede afectar a los resultados y los rendimientos en los test pueden verse afectados por una gran variedad de factores ( toma de psicofármacos, ansiedad, déficits sensoriales, nivel cultural premórbido, etc. ).

La necesidad de una orientación más ecológica en la evaluación neuropsiocológica de las funciones ejecutivas ha dado relevancia a que junto a la identificación de los principales procesos cognitivos implicados resulta esencial la identificación del impacto de estos problemas en los aspectos funcionales de la vida diaria y la determinación de la capacidad que tiene el individuo para llevar una vida independiente, autónoma o sus recursos personales para integrarse en una actividad profesional normalizada.

Hemos de reconocer que en los últimos años se viene desarrollando nuevos test y pruebas especialmente diseñadas para intentar valorar con más finura estos déficits ejecutivos. Se pueden citar entre otras el test de evaluación conductual del sindrome disejecutivo, el test de selección de clases, tareas de ejecución dual, test de preferencias, tareas de juego, tareas de planificación financiera, pruebas de cambio o el test de compe- tencia cognitiva. Además de estas pruebas hoy en día existe un gran consenso entre los profesionales en la necesidad de utilizar cuestionarios fenomenológicos que aporten información sobre el funcionamiento de estos pacientes; entre estos cuestionarios nosotros aconsejariamos la escala de Iowa modificada y la Neurobehavioral Rating Scale (NRS) (Muñoz-Cespedes, Miguel Tobal, 2000). Cabe destacar asimismo la necesidad de interpretar con cautela los datos proporcionados por las pruebas de autoinforme o los cuestionarios. La dificultad para comprender la complejidad de algunas afirmaciones $\mathrm{y}$, sobretodo, la limitada capacidad de autoconciencia constituyen dos capacidades centrales asociadas con las funciones del córtex prefrontal lo que puede afectar a la fiabilidad y a la validez de la información que proporcionan dichos cuestionarios.

A modo de sugerencias podemos plantear las siguientes recomendaciones: a) los resultados de los test y baterias neuropsicológicas deben considerarse como elementos complementarios e integrarlos en un marco comprensivo, b) la selección de los instrumentos de exploración neuropsicológica deben basarse en su capacidad para ofrecer información sobre los mecanismos subyacentes alterados, en su validez ecológica y deben ser sensibles a los avances que se producen y c) la evaluación neuropsicológica debe llevarse a cabo por personas especializadas que interpreten los datos en función de un corpus de conocimiento sólido sobre las relaciones entre cerebro y conducta (Tirapu, Muñoz Céspedes, Pelegrin, 2002).

Cripe (1996), en un magnífico capitulo sobre validez ecológica de los test neuropsicológicos que miden los déficits ejecutivos elabora una lúcida reflexión sobre lo que el denomina "the mind data problem" sugiriendo que la dificultad para medir el funcionamiento ejecutivo 
es un problema metafísico y epistemológico ya que las puntuaciones en los test son meras representaciones simbólicas reduccionistas. Los presupuestos básicos de Cripe son los siguientes:

1. Objetos estáticos y simples pueden ser medidos con un razonable grado de fiabilidad.

2. Las medidas no son el objeto. Son una representación simbólica del objeto.

3. Cuando los objetos estáticos son más complejos en su diseño y estructura la medida es más dificultosa.

4. Los objetos en movimiento son más difíciles de medir.

5. Múltiples objetos y realidades en continuo movimiento e interactuando en un sistema dinámico son muy dificiles de medir y describir.

6. Cuando más complejas son las realidades que deseamos medir la fiabilidad es más alta si empleamos múltiples medidas.

7. Reducir una realidad compleja y dinámica a pequeñas realidades incompletas nos aparta de la comprensión de la realidad global.

8. La realidad es más fácil de comprender cuanta más información y de más informadores obtengamos.

9. Los simples resultados en un test excluyen mucha información acerca de los procesos subyacentes en la conducta.

10. $\mathrm{El}$ acto mental como un producto de una interacción dinámica de múltiples y complejos sistemas dinámicos será mejor conocido cuanta más información seamos capaces de recabar y de integrar en un modelo comprensivo. 


\section{BIBLIOGRAFÍA}

Acker M.B: A review of the ecological validity of neuropsychological test. En Tupper D.E.

Cicerone, K.D. (1990). The neuropsychology of every day life: assesment and basic competences. Kluver Academic Publishers.

Anderson, S.W., Damasio, H., Dallas Jones, R., Tranel, D. (1991). WCST perfomance as a measure of frontal lobe damage. J. Clinic. Experim. Neuropsycol. 13(6). 909-922.

Baddeley, A., Della Sala, S., Papagno, C., et al. (1998). Dual-task performance in dysexecutive and nondysexecutive patients with a frontal lesion. Neuropsychology. 11(2). 187-194.

Bechara, A., Damasio, A.R, Damasio, H., Anderson, S.W. (1994). Insensitivity to future consequences following damage to human prefrontal cortex. Cognition. 50.7-15.

Berrios, G.E., Quemada, J.I. (1995). La neuropsiquiatría del daño cerebral traumático: aspectos conceptuales. Monografias de psiquiatría. Año VII (6).3-11.

Carter, S.C., Mathew, M.B., Cohen J.D. (1999) The contribution of the anterior cingulate cortex to executive processes in cognition. Reviews in Neuroscience, $10.49-57$

Cripe, L.I. (1996). The ecological validity of executive function testing. En Sbodorne R.J., Long Ch. J.: Ecological validity of neuropsychological testing. GR Press. Lurie Press. Florida.

Cummings, J.L. (1993). Frontal subcortical circuits and human behavior. Arch Neurol, 50. 873 880 .

Damasio, A.R. (1998). The somatic marker hypothesis and the possible functions of the prefrontal cortex. En:Roberts A.C., Robbins T.W. Weiskrantz L.:The frontal cortex: Executive and cognitive functions. Oxford University Press.

Damasio, A.R., Tranel, D., Damasio, H. (1990). Individuals with sociopathic behavior caused by frontal damage fail to respond autonomocally to social stimuli. Behavioral Brain Research. 41.81-94.

Damasio, A.R., Tranel, D., Damasio, H. (1991). Somatic markers and the guidance of behavior:
Theory and preliminary testing. En Levin H.S. Eisenberg H.M:, Benton A:L:: Frotal lobe function and dysfunction. Oxford University Press.

Damasio, A.R. (1994). Descartes' Error. Emotion, reason and the human brain. Putnam's Sons. New York.

Damasio, A.R:, Damasio, H. (1995). Cortical systems for retrieval of concrete knowledge: the convergence zone framework. En Koch C.: Largescale neuronal theories of the brain. MIT Press. Cambridge.

Ellis, A.W., Young, A.W. (1992). Neuropsicologia cognitiva humana. Masson.

Eslinger, P.J., Damasio, A.R. (1985). Severe disturbance of higher cognition after bilateral frontal lobe abltion: patient EVR. Neurology. 35 1731-1741.

Faglioni, P. (1999). The frontal lobe. En Denes G., Pizzamiglio L.: Handbook of clini9cal and experimental neuropsychology. Psychology Press. Taylor \& Francis.

Fodor, J.A. (1983). The modularity of mind. Mass. MIT Press. Cambridge.

Fuster, J.M. (1997). The prefrontal cortex: Anatomy, psyssiology and neuropsychology of the frontal lobe. Lippincott.Raven. New York.

Goel, V., Grafman, J., Tajik, J., et al. (1997). A study of the performance of patients with frontal lobe lesions in a financial planning task. Brain. 120. 1805-1822.

Goldberg, E., Podell, K. (2000). Adaptive decision making, ecological validity and the frontal lobes. J. Clinic. Experimen. Neuropsycholoy. 22(1). 56-68.

Golden, C.H. (1995). Stroop colour and word test. A manual for clinical and experimental use. Stoelting. Chicago.1974. Edición Española TEA.

Hall, K.M. (1997). The Functional Assessment Measure (FAM). J Rehabil Outcomes. 1(3). 63-65.

Heaton, R.K., Chelune, G.J:, Talley, J.L., et al. (1993). Wiscijonsin Card Shorting Test. Psychological Assesment Resources. Odessa. Florida. Edición española TEA.1997. 
Jones-Gottman, Milner (1977). Design Fluency test: the invention of nonsense drawings after focal cortical lesions. Neuropsychologia. 15. 653-674.

Levin, H.S., High, W.M., Goethe, K.E., et al (1987) The neurobehavioral rating scale: assesment of the behavioral sequelae of head injury by the clinician. J. Neurol. Neurosurg. Psichyatry. 50. 183-193.

Mesulam, M.M. (1986). Frontal cortex and behavior. Annals of Neurology. 19. 320-325

Montgomery, G.K. (1995). A multi-factor account of disability after brain nijury: implications for neuropsychological counselling. Brain Injury. 9(5). 453-469.

Mora, F. (1995). El problema cerebro-mente. Alianza Editorial.

Muñoz Cespedes, J.M., Miguel Tobal, J.J., Cano, A. (2000). Evaluación de las alteraciones emocionales en personas con TCE. Psicothema. 12 (1). 99-106

Muñoz Céspedes, J.M., Tirapu Ustárroz, J. (2001). Rehabilitación Neuropsicológica, Madrid. Sintesis.

Nemerof, C.B., Clinton, D.K., Berns, G.S (1999). Functional brain imaging: Twenty-first century phrenology or psychobiological advance for the millennium?. Am. J. Psychiatry. 156(5). 671673
Pelegrin Valero, C. (1995). Neuropsiquiatría del daño prefrontal en los TCE: Validación española de la entrevista de IOWA. Tesis Doctoral. Facultad de Medicina. Zaragoza.

Quintana, J., Fuster, J.M. (1999). From perception to action: Temporal integrative functions og prefrontal and parietal neurons. Cerebral Cortex. 9. 213-221.

Reitan, R.M. (1958). Validity of the TMT as an indicator of organic brain damage. Percept. Motor Skills. 271-276.

Rhimer, Z. (1999). Dysthymic disorder: implications for diagnosis and treatment. Current Opinion of Psychiatry. 12(1). 69-75.

Saver, J.L., Damasio, A.R. (1991). Preserved access and processing of social knowledge in a patient with adquired sociopathy due to ventromedial frontal damage. Neurospychologia. 29 (12). 1241-1249.

Shallice, T. (1982). Specific impairments in planing. Phil. Trans. Royal Soc. 298. 199-209.

Thurstone. (1962). Thurstone Word Fluency Test. Science Research Associates.

Tirapu Ustárroz, Muñoz Céspedes, Pelegrin Valero (2002). Funciones ejecutivas: necesidad de una integración conceptual. Rev Neurol. 